\title{
BMJ Open Delirium prevention and treatment in the emergency department (ED): a systematic review protocol
}

\author{
Elijah Blue Dahlstrom (D) , ${ }^{1}$ Jin Ho Han, ${ }^{2}$ Heather Healy, ${ }^{3}$ Maura Kennedy, ${ }^{4}$ \\ Glenn Arendts, ${ }^{5}$ Jacques Lee, ${ }^{6}$ Chris Carpenter, ${ }^{7}$ Sangil Lee ${ }^{8}$
}

To cite: Dahlstrom EB, Han $\mathrm{JH}$, Healy $\mathrm{H}$, et al. Delirium prevention and treatment in the emergency department (ED): a systematic review protocol. BMJ Open 2020;10:e037915. doi:10.1136/ bmjopen-2020-037915

- Prepublication history and additional file for this paper is available online. To view these files, please visit the journal online (http://dx.doi.org/10. 1136/bmjopen-2020-037915).

Received 21 February 2020 Revised 30 August 2020 Accepted 10 September 2020

Check for updates

(C) Author(s) (or their employer(s)) 2020. Re-use permitted under CC BY-NC. No commercial re-use. See rights and permissions. Published by BMJ.

For numbered affiliations see end of article.

Correspondence to

Sangil Lee;

sangil-lee@uiowa.edu

\section{ABSTRACT}

Introduction Delirium is a dangerous syndrome of acute brain dysfunction that is common in the emergency department (ED), especially among the geriatric population. Most systematic reviews of interventions for delirium prevention and treatment have focused on inpatient settings. Best practices of effective delirium care in ED settings have not been established. The primary objective of this study is to identify pharmacologic and non-pharmacologic interventions as applied by physicians, nursing staff, pharmacists and other ED personnel to prevent incident delirium and to shorten the severity and duration of prevalent delirium in a geriatric population within the ED.

Methods and analysis Searches using subject headings and keywords will be conducted from database inception through June 2020 in MEDLINE, EMBASE, Web of Science, PsychINFO, CINAHL, ProQuest Dissertations and Theses Global and Cochrane CENTRAL as well as grey literature. Database searches will not be limited by date or language. Two reviewers will identify studies describing any interventions for delirium prevention and/or treatment in the ED. Disagreements will be settled by a third reviewer. Pooled data analysis will be performed where possible using Review Manager. Risk ratios and weighted difference of means will be used for incidence of delirium and other binary outcomes related to delirium, delirium severity or duration of symptoms, along with $95 \%$ Cls. Heterogeneity will be measured by calculating $P$, and a forest plot will be created. If significant heterogeneity is identified, metaregression is planned using OpenMeta to identify possible sources of heterogeneity.

Ethics and dissemination This is a systematic review of previously conducted research; accordingly, it does not constitute human subjects research needing ethics review. This review will be prepared as a manuscript and submitted for publication to a peer-reviewed journal, and the results will be presented at conferences.

PROSPERO trial registration number CRD42020169654.

\section{INTRODUCTION}

Delirium is an acute confusional state characterised by declines in attention, awareness and cognition. Fluctuations of mental status over time are characteristic and necessary for diagnosis. ${ }^{12}$ Delirium is common in the acute care setting including the emergency

\section{Strengths and limitations of this study}

This study will utilise three reviewers to analyse articles and resolve discrepancies of data collection.

- Study will be conducted with a well-defined search strategy including a search of unpublished and grey literature.

- Study will search a broad group of databases to identify studies produced by physicians, nurses, pharmacists and other researchers.

- There will be increased heterogeneity due to the large number of interventions included.

- Some outcomes, such as delirium duration, may be assessed differently in each study, limiting the analysis.

department (ED), and is particularly prevalent among adults older than 65 years. As many as $7 \%-17 \%$ of older adults who present to the ED meet diagnostic criteria for delirium. ${ }^{3-9} \mathrm{ED}$ providers miss delirium in up to $80 \%$ of cases. ${ }^{6}$ ED delirium is associated with significantly increased in-hospital, 30-day and 6-month mortality, as well as loss of independence, ${ }^{5-11}$ accelerated cognitive decline and post-traumatic stress disorder which are especially troubling to patients and their families. ${ }^{11} 12$ Delirium can be characterised by motor subtypes-namely hypoactive $(92 \%)$, hyperactive or mixed delirium $(8 \%){ }^{4}$ Our understanding of delirium prognosis is evolving through a growing body of literature exploring associations between classification of delirium aetiology, motor subtypes and severities of outcomes. ${ }^{41314}$

Interventions aimed at reducing the incidence and severity of delirium have been studied in various settings, but our preliminary searches revealed a paucity of ED research. $^{15}$ Multicomponent prevention programmes in hospitalised patients showed a reduction in delirium incidence. ${ }^{1617}$ Several Cochrane reviews have been published since 2012, which reported the utility 
of pharmacological agents, ${ }^{18}{ }^{19}$ delirium prevention programmes in hospital setting ${ }^{16}$ and long-term care. ${ }^{20}$ Thus, both pharmacologic and non-pharmacologic interventions applied by physicians, nursing staff, pharmacists and other ED personnel could have a role in reducing the incidence, severity or duration of delirium in the $\mathrm{ED}$, as reported in the studies from other practice settings including inpatient medical, surgical and palliative care units and long-term care facilities. ${ }^{16-19}$ 21-24 The ED is a critical setting for delirium screening and preventative measures, since more than half of geriatric hospitalisations begin in the ED. ${ }^{25}$

Clearly identifying, preventing and treating delirium is critical for improved patient care. Recognising the importance of improved delirium evaluation, management and prevention, the American College of Emergency Physicians developed an electronic tool, the ADEPT tool, to help improve care provided of older adults with or at risk of delirium in the ED. ${ }^{26}$ ADEPT stands for Assess, Diagnose, Evaluate, Prevent, and Treat, and is used as a checklist to aid in the care of the confused and agitated elderly patient. The purpose of this study is to better understand which delirium prevention or treatment strategies provide the most compelling evidence of effectiveness and feasibility in ED settings.

\section{Objective of systematic review and meta-analysis}

This review aims to identify pharmacologic and nonpharmacologic interventions as applied by physicians, nursing staff, pharmacists and other ED personnel to prevent incident delirium and to shorten the severity and duration of prevalent delirium in a geriatric population within the ED.

\section{METHODS AND ANALYSIS}

\section{Protocol design and registration}

The study is a systematic review and meta-analysis to summarise randomised controlled trials and quasiexperimental studies. The study protocol adheres to the Preferred Reporting Items for Systematic Reviews and Meta-analysis Protocols (PRISMA-P; see online supplemental table 1). ${ }^{27}$

\section{Study characteristics}

This review will consider quasi-experimental or randomised controlled trial designs that evaluate the effectiveness of a prevention or treatment intervention(s) for all forms of delirium (ie, hypoactive, hyperactive and mixed subtypes), evaluating incidence and severity of delirium as primary outcomes. This review will exclude studies solely focused on delirium tremens or emergence delirium, but will not exclude studies that include these diagnoses as part of a broader delirium definition. Published studies, studies that are pending publication, and unpublished studies will be included to reduce the bias of selective outcome reporting. Further inclusion and exclusion criteria are listed in table 1.

\section{Types of participants}

This systematic review will include studies where participants are aged 65 or older who present initially to the ED and are evaluated for delirium. We will include studies that use subjects under the age of 65 if they also report data on people over age 65 .

\section{Types of interventions}

Interventions of interest are those aimed at prevention or treatment of delirium. Prevention will be defined as any

\begin{tabular}{|c|c|c|}
\hline $\begin{array}{l}\text { PICOS } \\
\text { strategy }\end{array}$ & Inclusion criteria & Exclusion criteria \\
\hline Intervention & $\begin{array}{l}\text { Multicomponent interventions, single component non-pharmacologic } \\
\text { interventions performed by physicians, nursing staff and pharmacists, } \\
\text { pharmacologic interventions performed by physicians, nursing staff and } \\
\text { pharmacists }\end{array}$ & None \\
\hline Comparator & Usual care & None \\
\hline Outcomes & $\begin{array}{l}\text { Primary outcomes: } \\
\text { Incidence of delirium (prevention study) } \\
\text { Severity of delirium (treatment study) } \\
\text { Secondary outcomes (treatment study): } \\
\text { Delirium duration } \\
\text { In-hospital and long-term mortality, discharge to skilled nursing facility, long- } \\
\text { term quality of life, functional status and long-term cognition }\end{array}$ & $\begin{array}{l}\text { Absence of the use of } \\
\text { validated delirium assessment } \\
\text { tool for delirium diagnosis or } \\
\text { severity }\end{array}$ \\
\hline Study design & Randomised controlled trials, quasi-experimental studies & None \\
\hline
\end{tabular}

ADEPT, Assess, Diagnose, Evaluate, Prevent, Treat; DSM, Diagnostic and Statistical Manual of Mental Disorders; GRADE, Grades of Recommendation Assessment, Development and Evaluation; ICD, International Classification of DIsease; PICOS, Population, Intervention, Comparison, Output, Study. 
method employed specifically to reduce the incidence of delirium. Treatment will be defined as any treatments aiming to reduce the severity or duration of diagnosed delirium. Both single-component and multicomponent non-pharmacologic interventions will be considered for this systematic review. All pharmacologic interventions such as typical and atypical antipsychotics, benzodiazepines, alpha-2 agonists, sedatives, opioids, cholinesterase inhibitors, melatonin and melatonin receptor agonists will be considered for inclusion. Single-component and multicomponent non-pharmacologic interventions as well as pharmacologic interventions will be included if performed by a physician, nurse, pharmacist or other ED staff.

\section{Types of outcome measures}

For all outcome measures, delirium should be defined and assessed in triage, ED or inpatient by a validated or acceptable diagnostic tool for delirium reported in the peer-reviewed journal, including Single Question to Identify Delirium, Ultrabrief two-item Bedside Test, Delirium Triage Screen, 4AT, brief Confusion Assessment Method, applicable diagnostic tools listed on the Delirium Severity Measures Summary Table developed by the Network for Investigation of Deliurium: Unifying Scientists (NIDUS), ICD 10 criteria and DSM IV/V diagnosis. ${ }^{28}{ }^{29}$ Delirium severity will be measured initially in triage or the ED using any of the existing tools defined in the Delirium Severity Measures Summary Table developed by NIDUS, or as defined in the specific study. ${ }^{29}$ The primary outcomes will be incidence of delirium for any prevention study and severity of delirium for any treatment study.

Secondary outcomes for any treatment study will include delirium duration. Duration will be defined using each study's respective definition of duration, and or measured as first instance of multiple consecutive positive tests until time of first instance of multiple consecutive negative tests for studies that include such data. We will also include all-cause mortality, in-hospital mortality, level of care, discharge to skilled care facility, any type of fall, quality of life and long-term cognitive impairment. In-hospital mortality will be defined as any death while in the ED or during the initial admission. Level of care will be defined categorically as discharge to home, admission to a step-down unit, or admission to an intensive care unit. Discharge to a skilled care facility will be assessed as new placement in a skilled nursing home immediately following the initial ED visit and primary hospital admission, but not including home health nursing. Long-term quality of life, functional status, and long-term cognition will be included as a secondary outcome if assessed using a validated, non-disease specific tool (such as the Activities of Daily Living Questionnaire).

\section{Types of studies}

Both randomised controlled trials and quasi-experimental studies will be included.
Timing

We will use timing parameters that extend throughout the hospital stay and up to a 180-day follow-up.

\section{Setting}

This review will be restricted to studies where initial delirium assessment starts in the ED or in triage and subsequent intervention(s) starts in triage, the ED or inpatient wards. This is because more than $50 \%$ of older adults who required hospitalisation are admitted through the ED, and effective screening, treatment and delirium prevention programme can have a significant impact on the remainder of their hospital stay. ${ }^{25}$

\section{Information sources}

Strategies for searching the literature will be developed to locate studies relating to the concepts; delirium, ED, interventions and the geriatric population. The strategies will use subject headings and keywords. A librarian (HH) will conduct searches in MEDLINE (Ovid), EMBASE (Elsevier), Web of Science, PsycINFO (EBSCOhost), CINAHL, ProQuest Dissertations and Theses Global and Cochrane CENTRAL (Wiley). The search strategies will not be limited by language. Each database will be searched from database inception through June 2020. The team will supplement the electronic database searches by looking for trial protocols and ongoing studies through ClinicalTrials.gov and PROSPERO. Additional unpublished sources and conference abstracts will be sought out directly from conference proceedings and primary authors. For each included article, the reference list will be searched for additional relevant studies.

\section{Search strategy}

The specific search strategies for each database will be developed by a health sciences librarian (HH) trained in systematic review searching. The team will provide input on the terminology for the strategies, and the strategies will be peer reviewed, using the PRESS guideline, by another health sciences librarian. ${ }^{30} \mathrm{~A}$ draft PubMed search strategy follows in online supplemental file.

Clinicaltrials.gov and PROSPERO will be searched for incomplete or recently completed studies. As the paucity of literature exists in the prevention and intervention of delirium in the ED, we will identify any preliminary or unpublished study through the content experts $(\mathrm{JHH}$, MK, GA and JL).

\section{Data management}

Database search results will be transferred to EndNote and deduplicated using the published methodology for deduplication as laid out by Bramer, Giustini and de Jonge. ${ }^{31}$ Citations will be stored and sorted using Endnote. Data will be compiled and meta-analysed using Review Manager (RevMan, V.5.3; The Nordic Cochrane Centre, The Cochrane Collaboration). OpenMeta (Analyst) (Center for Evidence-Based Medicine, Brown School of Public Health) will be used for regression with continuous covariates. 


\section{Selection process}

This review will follow the PRISMA standards for reporting a systematic review and meta-analysis. ${ }^{27}$ Studies identified via the search strategy and other methods will undergo an initial review by two independent reviewers who will review the title and abstract to determine whether the study meets the defined inclusion criteria. Studies where there is disagreement between the reviewers will be included in the secondary review.

Selected studies will subsequently be subjected to fulltext review, again with two independent reviewers utilising inclusion and exclusion criteria. A third reviewer will settle disagreements. We will report the kappa to measure inter-rater reliability. This analysis will be documented in a PRISMA flow diagram, and the process will result in a final list of included articles.

\section{Data collection}

Reviewers will use a standardised data collection form to gather desired information (see online supplemental table 2). The form will be developed following the style outlined by 'Data collection form for intervention review-RCTs and non-RCTs' from the Cochrane Collaboration. ${ }^{32}$ Data collection will be done independently. Calibration exercises will be used prior to conducting the review to ensure that each reviewer is consistent with their data collection. A third reviewer will check for discrepancies between data sheets for each given article and settle any disagreements. We will reach out to original study authors for details or data if any uncertainties arise.

\section{Data items}

We will collect data on the year of publication, study design, method of evaluating for delirium or delirium severity, and interventions for delirium prevention and/ or treatment. We will extract data on outcomes, including incidence of delirium, duration of delirium, delirium severity, mortality (30-day and in-hospital), new admission to a skilled nursing facility after ED visit, admission to an intensive care unit, quality of life and persistent cognitive deficits for intervention and control groups.

\section{Risk of bias assessment}

The study will use the Cochrane risk of bias assessment tool for randomised clinical trials and New-Castle-Ottawa scale for quasi-experimental studies for quality assessments. ${ }^{33-35}$ Direct quotes from each study will be used for all subsections of the tool in order to assess the risk of bias. Two independent reviewers will assess risk of bias, and the third reviewer will settle any discrepancy. We will report kappa to report the inter-rater agreement. The quality of evidence for an association between treatment, prevention and delirium-related outcomes will be evaluated in accordance with the GRADE system. ${ }^{36}$ This system grades quality of evidence at four levels: high (4), moderate (3), low (2) and very low (1). For high evidence, the requirements are a randomised, double-blinded study design with no selection biases. We will assess publication bias using funnel plots.

\section{Data synthesis}

If the studies, or an adequate subset of the studies in the review have sufficiently homogeneous outcomes data, meta-analysis will be conducted. Data associated with primary and secondary outcomes will be analysed in aggregate. For dichotomous data, risk ratios will be calculated with $95 \%$ CIs. A weighted difference of means will be used for continuous data with $95 \%$ CIs. Heterogeneity will be assessed using demographic features (age, sex) as well as study design factors (such as treatment type), and an $I^{2}$ value will be calculated. A value of less than $50 \%$ will be considered sufficiently homogeneous. ${ }^{37}$ Forest plots will be created using this data. Individual outcomes will be combined and calculated using RevMan V.5.3. ${ }^{38}$

\section{Subgroup analysis}

Subgroup analysis will be conducted based on type of intervention (pharmacologic and non-pharmacologic), delirium motor subtype (hyperactive, hypoactive, mixed), delirium severity, presumed delirium aetiology (phenotype) and whether incident or prevalent delirium was studied. Possible sources of heterogeneity tested includes age, antipsychotics, delirium subtype and study year. A linear metaregression model weighted to reflect the variance of the individual studies will be used to model the data.

\section{Patient and public involvement}

Patients and the public were not directly involved in the development of the research question or the design of the study, however many of the outcome measures (including quality of life, level of care and functional status) were chosen specifically to study the impact of delirium prevention and treatment measures on the patient experience. This study was conducted without direct patient involvement and patients were not involved in patient recruitment or conduction of the study.

\section{Amendments}

Amendments to this protocol, particularly database searches, will be documented in PROSPERO.

\section{ETHICS AND DISSEMINATION}

There will be no human subject participants involved in this review. The results of this review will be submitted for publication to a peer-reviewed journal. Other dissemination may include presentations at conferences and seminars.

\section{DISCUSSION}

Delirium is common and serious geriatric syndrome for which prevention and treatment best practices have not been established in the ED setting. ${ }^{39}$ One of the strengths of this study is that it will compile the relevant evidence for delirium prevention and intervention within the ED setting to identify any effective strategy. Another strength 
Table 2 A list of Cochrane reviews examining delirium intervention since 2012

\begin{tabular}{|c|c|c|}
\hline Study author/year & Main intervention & Setting* \\
\hline $\begin{array}{l}\text { Woodhouse et al } \\
(2019)^{20}\end{array}$ & $\begin{array}{l}\text { Software-based } \\
\text { identification of high-risk } \\
\text { medication }\end{array}$ & Long-term care \\
\hline Burry et al (2018) $)^{40}$ & Antipsychotic agent & $\begin{array}{l}\text { Non-ICU inpatient } \\
\text { ward }\end{array}$ \\
\hline $\begin{array}{l}\text { Herling et al } \\
(2018)^{41}\end{array}$ & $\begin{array}{l}\text { Delirium prevention study } \\
\text { (pharmacological) }\end{array}$ & ICU \\
\hline Yu et al $(2018)^{19}$ & Cholinesterase inhibitor & $\begin{array}{l}\text { Non-ICU inpatient } \\
\text { ward }\end{array}$ \\
\hline $\begin{array}{l}\text { Punjasawadwong } \\
\text { et al (2018) }\end{array}$ & Electroencephalogram & Perioperative \\
\hline Siddiqi et al (2016) ${ }^{16}$ & $\begin{array}{l}\text { Delirium prevention study } \\
\text { (pharmacological and non- } \\
\text { pharmacological treatment) }\end{array}$ & $\begin{array}{l}\text { Mixed setting } \\
\text { (none ED) }\end{array}$ \\
\hline Candy et al (2012) ${ }^{18}$ & Pharmacological therapy & $\begin{array}{l}\text { Delirium with } \\
\text { AIDS in the } \\
\text { palliative care }\end{array}$ \\
\hline
\end{tabular}

*We were unable to identify any review in the ED.

ED, emergency department; ICU, intensive care unit.

of this study is that it will be conducted using a welldefined search strategy. We will consult transdisciplinary delirium for grey literature, unpublished works and ongoing studies to minimise the risk of bias of selective outcome reporting.

The review will explore a new insight to prevention and treatment options to employ in the ED, which have become the main entrance for the majority of older adults to the hospital. The clinical care delivered in the ED influences downstream clinical care and therefore, may have a significant impact on outcomes. We are aware of a total of seven Cochrane reviews published since 2012 that examine the various delirium prevention and treatment interventions in varied clinical settings (table 2). ${ }^{16} 18-2040-42$

One Cochrane review examined the effect of antipsychotic agents for delirium treatment in non-ICU hospitalised patients and found no impact on delirium severity or duration. ${ }^{40}$ Due to limited research in the palliative care setting, evidence was deemed insufficient to assess the impact of pharmacologic therapies for the treatment of delirium in terminally ill patients. ${ }^{18}$ Similarly, the data were deemed insufficient to evaluate the effect of cholinesterase inhibitors in the treatment of delirium in the non-ICU hospital setting. A review evaluating prevention strategies in long-term care settings found limited evidence on interventions for preventing delirium, but a software-based intervention to identify high-risk medications and a pharmacist-led medication review reduced the incidence of delirium. ${ }^{20}$ There is strong evidence that a multicomponent delirium prevention programme reduces the incidence of delirium in the non-ICU hospital setting. Despite the ample number of systematic reviews, it is important to note that none of these focused on delirium interventions in the ED (table 2).

Our study protocol may have limitations, including increased heterogeneity due to the large number of different interventions being included. There may also be limitations to the statistical power of our findings depending on the quantity of literature in this setting. Although we set a comprehensive list of outcomes, it may not be possible to find any studies that list them, for example, quality of life.

The importance of delirium prevention and management in the ED setting is underscored by the inclusion of delirium recognition and management as a best practice in the Geriatric ED Guidelines, ${ }^{43}$ a quality indicator for geriatric emergency care, ${ }^{44}$ a core competency for emergency medicine residents ${ }^{45}$ as well as by the recent dissemination of the ADEPT tool. ${ }^{26}$ Although guidelines and core competencies reflect the expert consensus-based best practices in EM, high-quality research evidence to support ED delirium prevention and intervention approaches have been lacking. This review will address a critical need to synthesise research into delirium prevention and management in the ED setting to further improve the care of older ED patients with or at risk for delirium and create a roadmap for future researchers. ${ }^{4647}$

\section{Author affiliations}

${ }^{1}$ Department of Emergency Medicine, University of lowa Roy J and Lucille A Carver College of Medicine, lowa City, lowa, USA

${ }^{2}$ Department of Emergency Medicine, Vanderbilt University, Nashville, Tennessee, USA

${ }^{3}$ University of lowa Libraries, University of lowa Roy J and Lucille A Carver College of Medicine, lowa City, lowa, USA

${ }^{4}$ Department of Emergency Medicine, Massachusetts General Hospital, Boston, Massachusetts, USA

${ }^{5}$ The University of Western Australia, Perth, Western Australia, Australia

${ }^{6}$ University of Toronto, Toronto, Ontario, Canada

${ }^{7}$ Department of Emergency Medicine, Washington University in Saint Louis, Saint Louis, Missouri, USA

${ }^{8}$ Department of Emergency Medicine, University of lowa Hospitals and Clinics, lowa City, Iowa, USA

\section{Twitter Sangil Lee @kagocchi28}

Contributors EBD is the first and SL is the corresponding author; EBD, CC and SL conceived and designed the study; HH designed the draft search strategy; SL, MK, JHH, JL, GA will acquire data; SL will analyse and interpret data; EBD and SL drafted the initial and final protocol; MK, JHH, JL, GA, CC, HH and SL performed critical revisions of the protocol. All authors approved the final version of the protocol.

Funding This study was supported by the departmental seed funding by the Department of Emergency Medicine at the University of lowa Hospitals and Clinics. The protocol was not influenced by the funding source. Award/Grant number is not applicable.

Competing interests CC: conflicts include SAEM Board of Directors, Geriatric Emergency Care Applied Research Network investigator leading cognitive impairment core, Clinician-Scientists Transdisciplinary Aging Research Leadership Core, Academic Emergency Medicine Deputy Editor-in-Chief, Journal of the American Geriatrics Society Associate Editor, Schwartz-Reisman Emergency Medicine Institute International Advisory Board Chair, and American Board of Emergency Medicine MyEMCert Editor.

Patient and public involvement Patients and/or the public were not involved in the design, or conduct, or reporting or dissemination plans of this research.

Patient consent for publication Not required. 
Provenance and peer review Not commissioned; externally peer reviewed.

Open access This is an open access article distributed in accordance with the Creative Commons Attribution Non Commercial (CC BY-NC 4.0) license, which permits others to distribute, remix, adapt, build upon this work non-commercially, and license their derivative works on different terms, provided the original work is properly cited, appropriate credit is given, any changes made indicated, and the use is non-commercial. See: http://creativecommons.org/licenses/by-nc/4.0/.

ORCID iD

Elijah Blue Dahlstrom http://orcid.org/0000-0002-0010-4235

\section{REFERENCES}

1 American Psychiatric Association. Diagnostic and statistical manual of mental disorders. 5th edn. Washington, DC: American Psychiatric Association, 2013.

2 Hosker CMG, Bennett MI. Delirium and agitation at the end of life. BMJ 2016;353:i3085.

3 Hustey FM, Meldon SW, Smith MD, et al. The effect of mental status screening on the care of elderly emergency department patients. Ann Emerg Med 2003;41:678-84.

4 Han JH, Zimmerman EE, Cutler N, et al. Delirium in older emergency department patients: recognition, risk factors, and psychomotor subtypes. Acad Emerg Med 2009;16:193-200.

$5 \mathrm{Han} \mathrm{JH}$, Shintani A, Eden S, et al. Delirium in the emergency department: an independent predictor of death within 6 months. Ann Emerg Med 2010;56-244-52.

6 Lewis LM, Miller DK, Morley JE, et al. Unrecognized delirium in ED geriatric patients. Am J Emerg Med 1995;13:142-5.

7 Suffoletto B, Miller T, Frisch A, et al. Emergency physician recognition of delirium. Postgrad Med J 2013;89:621-5.

8 Evensen S, Saltvedt I, Ranhoff $\mathrm{AH}$, et al. Delirium and cognitive impairment among older patients in Norwegian emergency departments. Tidsskr Nor Laegeforen 2019;139

9 Kennedy M, Enander RA, Tadiri SP, et al. Delirium risk prediction, healthcare use and mortality of elderly adults in the emergency department. J Am Geriatr Soc 2014;62:462-9.

10 Kakuma R, du Fort GG, Arsenault L, et al. Delirium in older emergency department patients discharged home: effect on survival. J Am Geriatr Soc 2003;51:443-50.

11 Hsieh SJ, Madahar P, Hope AA, et al. Clinical deterioration in older adults with delirium during early hospitalisation: a prospective cohort study. BMJ Open 2015;5:e007496.

12 Girard TD, Jackson JC, Pandharipande PP, et al. Delirium as a predictor of long-term cognitive impairment in survivors of critical illness. Crit Care Med 2010;38:1513-20.

13 Girard TD, Thompson JL, Pandharipande PP, et al. Clinical phenotypes of delirium during critical illness and severity of subsequent long-term cognitive impairment: a prospective cohort study. Lancet Respir Med 2018;6:213-22.

14 Devlin JW, Skrobik Y, Gélinas C, et al. Clinical practice guidelines for the prevention and management of pain, Agitation/Sedation, delirium, immobility, and sleep disruption in adult patients in the ICU. Crit Care Med 2018;46:e825-73.

15 Schrijver EJM, de Vries OJ, van de Ven PM, et al. Haloperidol versus placebo for delirium prevention in acutely hospitalised older at risk patients: a multi-centre double-blind randomised controlled clinical trial. Age Ageing 2018;47:48-55.

16 Siddiqi N, Harrison JK, Clegg A, et al. Interventions for preventing delirium in hospitalised non-ICU patients. Cochrane Database Syst Rev 2016;3:CD005563.

17 Nikooie R, Neufeld KJ, ES O, et al. Antipsychotics for treating delirium in hospitalized adults: a systematic review. Ann Intern Med 2019.

18 Candy B, Jackson KC, Jones L, et al. Drug therapy for delirium in terminally ill adult patients. Cochrane Database Syst Rev 2012;11:CD004770.

19 Yu A, Wu S, Zhang Z, et al. Cholinesterase inhibitors for the treatment of delirium in non-ICU settings. Cochrane Database Syst Rev 2018;6:CD012494.

20 Woodhouse R, Burton JK, Rana N, et al. Interventions for preventing delirium in older people in institutional long-term care. Cochrane Database Syst Rev 2019;4:CD009537.

21 Young J, Murthy L, Westby M, et al. Diagnosis, prevention, and management of delirium: summary of NICE guidance. BMJ 2010;341:c3704.

22 Marcantonio ER. In the clinic. delirium. Ann Intern Med 2011;154:ITC6-1, ITC6-2, ITC6-3, ITC6-4, ITC6-5, ITC6-6, ITC6-7,
ITC6-8, ITC6-9, ITC6-10, ITC6-11, ITC6-12, ITC6-13, ITC6-14, ITC615.

23 Marcantonio ER. Postoperative delirium: a 76-year-old woman with delirium following surgery. JAMA 2012;308:73-81.

24 Bergmann MA, Murphy KM, Kiely DK, et al. A model for management of delirious postacute care patients. J Am Geriatr Soc 2005;53:1817-25.

25 Greenwald PW, Estevez RM, Clark S, et al. The ED as the primary source of hospital admission for older (but not younger) adults. Am J Emerg Med 2016;34:943-7.

26 Shenvi C, Kennedy M, Austin CA, et al. Managing delirium and agitation in the older emergency department patient: the ADEPT tool. Ann Emerg Med 2020;75:136-45.

27 Shamseer L, Moher D, Clarke M, et al. Preferred reporting items for systematic review and meta-analysis protocols (PRISMA-P) 2015: elaboration and explanation. BMJ 2015;350:g7647.

28 Lee S, Gottlieb M, Mulhausen P, et al. Recognition, prevention, and treatment of delirium in emergency department: an evidence-based narrative review. Am J Emerg Med 2019.

29 Jones R. Delirium severity measures summary table. Network for investigation of Delirium: Unifying Scientists. Available: https:// deliriumnetwork.org/delirium-severity-measures-summary-table/ [Accessed 16 Oct 2019].

30 McGowan J, Sampson M, Salzwedel DM, et al. PRESS peer review of electronic search strategies: 2015 guideline statement. J Clin Epidemiol 2016;75:40-6.

31 Bramer WM, Giustini D, de Jonge GB, et al. De-duplication of database search results for systematic reviews in endnote. J Med Libr Assoc 2016;104:240-3.

32 Higgins J SG. Cochrane Handbook for systematic reviews of interventions version 5.1.0. The Cochrane collaboration, 2011.

33 Higgins JPT, Altman DG, Gøtzsche PC, et al. The Cochrane collaboration's tool for assessing risk of bias in randomised trials. BMJ 2011;343:d5928.

34 Wells G, Shea B, O'Connell D, et al. The Newcastle-Ottawa scale (NOS) for assessing the quality of nonrandomised studies in meta-analyses. Available: http://www.ohri.ca/programs/clinical_ epidemiology/oxford.asp [Accessed 30 Sep 2019].

35 Sterne JAC, Savović J, Page MJ, et al. RoB 2: a revised tool for assessing risk of bias in randomised trials. BMJ 2019;366:14898.

36 Guyatt G, Oxman AD, Akl EA, et al. GRADE guidelines: 1. Introduction-GRADE evidence profiles and summary of findings tables. J Clin Epidemiol 2011;64:383-94.

37 Higgins JPT, Thompson SG, Deeks JJ, et al. Measuring inconsistency in meta-analyses. BMJ 2003;327:557-60.

38 The Cochrane Collaboration. Review manager (RevMan) [Computer program]. Version 5.3. Copenhagen: The Nordic Cochrane Centre, 2014.

39 Han JH, Suyama J. Delirium and dementia. Clin Geriatr Med 2018;34:327-54.

40 Burry L, Mehta S, Perreault MM, et al. Antipsychotics for treatment of delirium in hospitalised non-ICU patients. Cochrane Database Syst Rev 2018;6:CD005594.

41 Herling SF, Greve IE, Vasilevskis EE, et al. Interventions for preventing intensive care unit delirium in adults. Cochrane Database Syst Rev 2018;11:CD009783.

42 Punjasawadwong Y, Chau-In W, Laopaiboon M, et al. Processed electroencephalogram and evoked potential techniques for amelioration of postoperative delirium and cognitive dysfunction following non-cardiac and non-neurosurgical procedures in adults. Cochrane Database Syst Rev 2018;5:CD011283.

43 Carpenter CR, Bromley M, Caterino JM, et al. Optimal older adult emergency care: introducing multidisciplinary geriatric emergency department guidelines from the American College of emergency physicians, American geriatrics Society, emergency nurses association, and Society for academic emergency medicine. Ann Emerg Med 2014;63:e1-3.

44 Terrell KM, Hustey FM, Hwang U, et al. Quality indicators for geriatric emergency care. Acad Emerg Med 2009;16:441-9.

45 Hogan TM, Losman ED, Carpenter CR, et al. Development of geriatric competencies for emergency medicine residents using an expert consensus process. Acad Emerg Med 2010;17:316-24.

46 Shenvi C, Wilson MP, Aldai A, et al. A research agenda for the assessment and management of acute behavioral changes in elderly emergency department patients. West J Emerg Med 2019;20:393-402.

47 Wilson MP, Shenvi C, Rives L, et al. Opportunities for research in mental health emergencies: Executive summary and methodology. West J Emerg Med 2019;20:380-5. 